\title{
A combined SP-RP model to estimate the value of improvements in freshwater angling in England
}

Paulo Rui Anciaes

University College London, United Kingdom

Paul Metcalfe

Antara Sen

PJM Economics

Accepted version of paper published in Journal of Environmental Economics and Policy, https://doi.org/10.1080/21606544.2019.1622454

\begin{abstract}
This paper estimates the value of improvements in the quality of freshwater angling sites in England, combining the results of stated preference (SP) models and a revealed preference (RP) model of the anglers' actual choices of fishing sites and number of trips over a season. The paper provides comprehensive information into what anglers value and how much, considering all fishery types (coarse, game, and mixed) and water body types (river, stillwater, and canal) and a wide range of fish species. The study also considers several locational characteristics of the fishing sites, which have seldom been included in either SP or RP studies. We found that anglers attach a substantial value to lack of pollution, availability of pegs, and an attractive site environment. On average, the maximum possible improvement in one of these attributes in a given site more than doubles the number of visitors to that site and generates a total additional benefit of more than $£ 10$ per existing trip. Increases in fish size and quantity are also predicted to cause considerable changes in the number of visits and additional benefit, especially when moving from small/low to medium levels.
\end{abstract}

Keywords: angling, water-based recreation, valuation, stated preference, choice modelling, revealed preference, linked random utility model 


\section{Introduction}

Angling, or recreational fishing, is one of the most popular sports in England, practiced by up to 4 million people (Simpson and Mawle 2010), spending an estimated total of around 30 million days per year in the activity (Radford et al. 2007). Given these numbers, angling is a particularly valuable economic and social activity. There is evidence that angling is also important in other countries such as Germany (Arlinghaus 2004), USA (Hughes 2015), Ireland (TDI 2013), and Scandinavian countries (Toivonen et al. 2004). However, in England as in other countries, angling is threatened by problems such as pollution and overfishing (Winfield 2016), which call for increased efforts in the strategic management and protection of fishing resources. This requires a better knowledge about the value that anglers attach to different aspects of the activity.

The value of improvements in the quality of angling sites can be estimated by calculating the anglers' willingness to pay for those improvements, using stated preference (SP) or revealed preferences (RP) methods. SP methods are based on choices made in hypothetical settings and so they are well-suited to provide valuations of future improvements. However, the use of hypothetical settings also limits the validity of the results. RP methods are based on anglers' actual behaviour (i.e. the choice of which sites to visit, and/or how often to visit them) but rely on information about recent fishing activity, and so they are limited in their ability to derive predictions with respect to future improvements, since the real world does not necessarily present the variation needed to explore all the possible types of improvements in the relevant characteristics of fishing sites.

This paper assesses the value of improvements in the quality of freshwater angling sites in England, combining the results of SP and RP methods. This approach allows us to exploit the strengths of the two approaches and estimate more robust and realistic valuations than possible when using either method in isolation. The SP data captures the anglers' choices over fishing site characteristics in a variety of hypothetical scenarios and the RP data grounds those choices with real-world choice behaviour (choice of fishing sites and number of trips over a year). The paper also contributes to the literature by considering attributes that are seldom included in either SP or RP studies, such as the locational characteristics of the fishing sites, and by integrating all fishery types (coarse, game, and mixed) and water body types (river, stillwater, and canal) and a wide range of fish species in the same analysis. 


\section{Literature review and contribution of the paper}

The economic value of angling is usually estimated by quantifying expenditures relating to angling, understood as indicators of the direct contribution of the activity to the economy (Peirson et al. 2001, PWC 2007, Butler et al. 2009, TDI 2013, Brown 2014, SQW 2015). In most cases, the indirect and induced economic effects of angling, measured in terms of increased income, are also quantified. This type of research usually involves surveys to river beat owners, fishing licence holders, or members of angling clubs, conducted in specific areas (at a catchment or river level), and producing values that are specific to those areas. However, Arlinghaus (2004), Radford et al. (2007) and TDI (2013) also produced estimates of the economic impact of angling for whole countries.

A different approach is to estimate the anglers' willingness to pay (WTP) for improvements in the quality of fishing sites, using SP methods. A number of studies have used contingent valuation, usually focusing on specific areas and often also on a single fish species (Berrens et al. 1993, Laitila and Paulrud 2006). An exception is the study of Toivonen et al. (2004), which compared values obtained in five different countries in Northern Europe. The number of studies using SP methods based on choice modelling has also been growing (Lawrence and Spurgeon 2007, Lee et al. 2013, Thangavelu et al. 2017). These studies usually elicit WTP for improvements in water quality and fish quantity and quality, with a few also considering accessibility to and congestion in the fishing site. However, they do not consider possible changes in visit numbers following improvements in the site attributes nor the resulting changes in consumer surplus. As such, they may underestimate the total benefit of the improvements. Paulrud and Laitila (2013) addressed this issue by modelling changes in number of fishing days as a function of the utility that can be derived from the available sites, for four different future scenarios regarding fish stocks.

The value of improvements in the quality of fishing sites can also be derived using RP approaches based on travel cost models. This approach can be applied at the level of individual sites, estimating willingness to pay per trip through models that relate the number of trips to a site with the travel costs to access it (Curtis 2002, Shrestha et al. 2002, Arismendi and Nahuelhual 2007, Hynes et al. 2015, Curtis and Stanley 2016, Wallentin 2016). It can also be applied at the level of the choice set of possible sites to visit, by using random utility models that relate the probability that a site is chosen with the characteristics of the site and the travel costs to access it. The choice of a site implicitly reveals how an angler trades off 
one site characteristic for another and the willingness to pay for changes in each characteristic (Train 1998, Murdock 2006, Mkwara et al. 2015, Melstrom et al. 2015).

However, the use of these two types of RP approaches in isolation gives only a partial view of the impact of changes in angling quality, which include both effects on site choice and on the number of trips made. This issue was addressed by Morey et al. (2002), who estimated a two-level linked model to value the impact of the reduction of fish stocks on the utility of existing trips and on the number of trips. Johnstone and Markandya (2006) extended this approach, by considering a wider range of attributes related to fish quantity and water quality.

A few studies have combined SP and RP data for valuing water quality in relation to recreational activities (Whitehead 2005, Eom and Larson 2006). However, fishing was usually treated as only one of several possible recreation activities and assessed only in terms of the water quality being suitable for fishing or not. Whitehead and Lee (2016) estimated a joint RP-SP model of recreational fishing behaviour but the model considered only the tradeoff between travel costs and stock abundance (measured by catch rates).

There is still little evidence, either from SP or RP applications, on the value of improvements in attributes other than the availability of specific species, fish quantity, and water quality. Some studies have found that fishing site characteristics such as availability of car parking, access for disabled anglers, and guiding services influence the number of trips made by anglers (Curtis and Breen 2017), which suggests that anglers attach value to these characteristics. However, there are no robust estimates of the magnitude of this value and of the trade-offs that anglers make between the different site characteristics.

This paper adds to this literature by providing up-to-date, nationally-representative, and comprehensive information into what anglers value and how much. In particular, the study makes three main contributions:

- It produces estimates of consumer surplus for hypothetical changes in fishery quality that are grounded in real-world behaviour (combining SP and RP methods) while considering both the added value for existing trips and the number and value of new trips.

- It derives values from changes in a large number of attributes relating to angling, including not only fish quality and quantity but also several locational characteristics of the site.

- It considers all fishery types (coarse, game, and mixed) and water body types (river, stillwater, and canal), as well as a wide range of fish species. 


\section{Overview of methods and structure of the paper}

The study consisted of two separate surveys, supporting SP and RP analyses of the anglers' preferences. The stated preference component of the study is presented in Section 4, which is split into two sub-sections, one for each of the two SP exercises. The first exercise (SP1) is a site choice exercise and provides information on the trade-offs between site attributes that anglers make when choosing a site. The second exercise (SP2) provides extra information on preferences for site attributes by asking anglers about the most and least important attributes when choosing a site. The two sub-sections of Section 4 are structured in the same way, first presenting the design of the SP exercise, followed by the specification of the choice model and the estimation results.

The revealed preferences component of the study is presented in Section 5 and includes a site choice model measuring the trade-offs between site attributes as well as a participation model measuring how trip frequency depends on the utility that anglers derive from the set of available sites. Section 5 is divided into three sub-sections, describing the RP data, methods, and results.

Section 6 brings together the SP and RP components by combining the SP1 and SP2 estimates and scaling them using the RP estimates. The RP site choice and participation models are then used to estimate the impact of site improvements on the number of visits to each site and on consumer surplus. Section 6 is divided into two sub-sections, describing methods and results.

\section{Stated preference}

The first stage of the research was an SP survey to estimate the value to anglers of changes in the characteristics of fishing sites. The survey consisted of two exercises, one focusing on choices between hypothetical site alternatives (SP1) and another asking participants about the most and least important attributes of a site when choosing where to go fishing (SP2). The attributes of both exercises were selected based on previous literature and discussions with stakeholders (UK Environmental Agency and the Angling Trust). The two exercises were linked through two common attributes: fish size and fish quantity. Besides the SP exercises, the survey also included questions about the characteristics of participants, such as gender, age, licence type (full, senior concession, disabled concession), and licence scope (annual or 
short term, trout/coarse or salmon/sea trout), as well as details about their most recent fishing trip (noted as 'base trip' in the analysis that follows).

The survey questionnaire was tested in a pilot survey of 95 anglers. The main stage of the survey then used a sample extracted from the UK Environment Agency's database of individuals residents in England, over 16 years old, and holding a rod licence (which is required for angling in England) at any time in 2016. A mixed-mode research design was used, including an online component (2,974 interviews), which allows a large sample to be obtained cost-effectively, and a Computer Assisted Telephone Interviewing (CATI) component with recruitment over the telephone (250 interviews), which corrects for biases inherent in a pure online approach, since not all anglers have easy access to the internet.

The sample had a predominance of men (97\%), individuals aged over $45(82 \%)$, holding a full licence (57\%), and an annual trout and coarse licence (80\%). The sample was not representative of the population of anglers, which has smaller proportions of individuals aged over $45(53 \%)$ and holding a full licence (47\%). For this reason, a raking (iterative proportional fitting) procedure was used to generate weights (Särndal 2007, Kott 2009). The procedure ensured that the known population totals matched the sample-weighted totals of men and women, age groups, and licence types and scopes. The weights were trimmed to the interval [0.25-4] to ensure that they were not excessively small or large for any of the participants (Théberge 2000). The interval was defined using a trial and error procedure, to ensure that the solution is accurate, i.e. the difference between population and weighted totals is minimal. The final calibrated weights were applied in the estimation of the SP models.

With regards to the participants' base trips, around two thirds (67\%) of participants visited stillwaters, $29 \%$ visited rivers, and $4 \%$ visited canals. More than three quarters $(76 \%)$ of participants fished coarse fish, $20 \%$ of participants fished trout, and $4 \%$ fished salmon.

\subsection{SP1: Trade-offs between site attributes}

\section{Design}

The SP1 exercise explored the trade-offs that anglers make between different site characteristics when choosing where to go fishing. The exercise consisted of eight questions (see an example in Figure 1) showing four alternative sites that could be chosen in the case of a fishing occasion. It was also possible to choose not to visit any of the sites. Participants were asked to state not only their most likely choice but also their least likely choice. This resulted in a substantial increase in data, and thereby statistical precision, for not much 
additional effort on the part of the participants. Using this approach, each choice situation could be split in three separate implicit choices: one indicating that the most preferred option was chosen from a choice set formed by the four options and the 'none of these' option, and the other two indicating that each of the middle two options (not the most or least preferred) was individually preferred to the least preferred option in a pairwise comparison.

\section{Figure 1: SP1 question format}

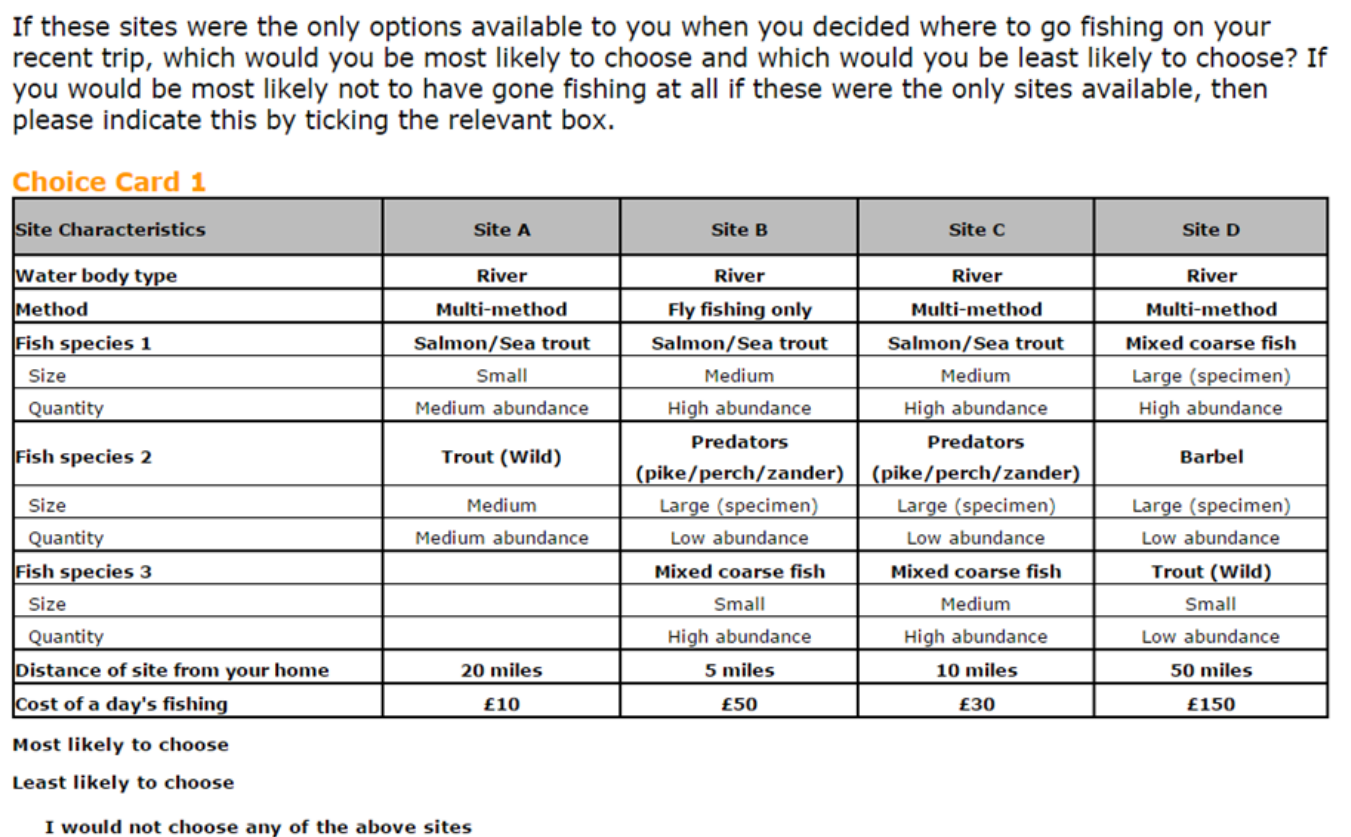

Table 1 shows the SP1 attributes and levels. Up to 3 different fish species were shown per site. The cost attribute measured the cost of obtaining permission to fish for a day. Participants were told that fish size and quantity were relative to the average for the species and that where a season permit was in place they should estimate the daily rate as the season permit cost divided by total trips to sites covered by the permit. In Options A and B, the water body types and the first species shown were always those in the participants' base trip.

Table 1: SP1 attributes and levels

\begin{tabular}{|c|c|}
\hline Attributes & Levels \\
\hline Water body type & River, Stillwater, Canal \\
\hline Fishing method & Fly-fishing only, Multimethod \\
\hline Fish species & $\begin{array}{l}\text { Trout (Wild), Trout (Stocked), Grayling, Salmon/sea trout, Predators } \\
\text { (pike/perch/zander), Barbel, Carp, Catfish, Mixed coarse fish }\end{array}$ \\
\hline Fish size & Small, Medium, Large \\
\hline Fish quantity & Low, Medium, High \\
\hline Distance from home (miles) & $1,2,5,10,15,20,30,50,100,200,300$ \\
\hline Cost of a day's fishing $(\mathfrak{£})$ & $0,5,10,15,20,25,30,40,50,60,75,100,125,150,200,250$ \\
\hline
\end{tabular}


The full list of restrictions applied to the choice set is shown in a table provided as supplementary information to the paper. The restrictions were based on discussions with the UK Environmental Agency and the Angling Trust and were applied to ensure that the scenarios shown would be plausible to participants. Distance was always below 100 miles if the distance of the base trip was below 30 miles and above 5 miles if the distance of the base trip was above 30 miles. This ensured that respondents would only choose between sites within a reasonable range of distances given the distance they travelled on their base trip. A set of 23 restrictions were specified in relation to the other attributes. As an example, the combination of canal and carp only occurred for low quantity and medium or large size. The frequency with which each fishing method was shown in Options A and B also depended on the species fished in the base trip. For example, fly-fishing appeared in $40 \%$ of the options when the species was trout, salmon/sea trout, or grayling, and never appeared in the case of other species. Each combination of water body type, fish species, size, and quantity was also associated with a particular subset of cost values. For example, canal, carp, and medium quantity and size was only associated with cost values of $£ 2, £ 5$, and $£ 10$. We assumed a skewed distribution within that subset of cost values, with the bulk of fisheries with low to middle values, fewer fisheries with very low values and even fewer fisheries with high values.

\section{Model}

The data was modelled using a mixed logit specification (McFadden and Train 2000), which allows for random taste variation across anglers, producing coefficients that vary over individuals. The utility $U_{i j}$ for an angler $i$ visiting a site $j$ on a given choice occasion is a function of the distance to the site $\left(d_{i j}\right)$, cost of a day's fishing $\left(c_{j}\right)$, other observed site characteristics $\left(x_{j}\right)$, and a random error term $\varepsilon_{i j}$ which accounts for all the unobserved characteristics that influence the angler's utility. $\beta_{i}$ is a vector of parameters to be estimated, assumed to have random and normal distributions over the population of anglers. The distance and cost parameters $\varphi$ and $\lambda$ were assumed to be fixed across anglers.

$$
U_{i j}=\varphi d_{i j}+\lambda c_{j}+\beta_{i} x_{j}+\varepsilon_{i j}
$$

The probability that angler $i$ chooses to visit site $j$ can be expressed as the probability that the utility associated with that site is greater than the utility associated with any other site $k$ in the angler's choice set.

$$
P\left(U_{i j}>U_{i k}\right)=P\left(\varphi d_{i j}+\lambda c_{j}+\beta_{i} x_{j}+\varepsilon_{i j}>\varphi d_{i k}+\lambda c_{k}+\beta_{i} x_{k}+\varepsilon_{i k}\right)
$$


If the error terms are independently and identically distributed with a Type I Extreme Value distribution, then it can be shown (McFadden 1974) that the probability $P_{i j}$ that angler $i$ chooses to visit site $j$ can be expressed in terms of a logistic distribution, as below. The parameters of the model can be estimated via maximum simulated likelihood.

$$
P_{i j}=\exp \left(\varphi d_{i j}+\lambda c_{j}+\beta_{i} x_{j}\right) /\left(\exp \left(\varphi d_{i j}+\lambda c_{j}+\beta_{i} x_{j}\right)+\Sigma_{k} \exp \left(\varphi d_{i k}+\lambda c_{k}+\beta_{i} x_{k}\right)\right)
$$

The dependent variable in the model was a dummy variable equal to 1 if a site alternative was chosen and 0 otherwise. The explanatory variables were the distance to the site, the cost of a day's fishing, dummy variables for the site characteristics (water body type, fishing method, fish species type, fish size and quantity) and a dummy variable representing the "would not choose any of the sites" option.

The willingness to pay (WTP) per trip for marginal changes in a site characteristic can be calculated from the estimated model as the ratio between the coefficient accounting for that characteristic and the coefficient of the cost variable. This ratio is an indicator of the trade-off that anglers make between the site characteristic and the cost of visiting the site (Hanemann 1984). Confidence intervals for WTP can be calculated using the Krinsky Robb parametric bootstrap method (Krinsky and Robb 1986).

\section{Results}

Table 2 shows the mean and standard deviations of the SP1 individual-level coefficients, and the WTP central values and 95\% confidence interval for each attribute level. As expected, on average anglers preferred sites closer to home, with lower permit costs, with larger sized and more quantity of fish. The WTP to go from small to medium and small to large size of fish was $£ 4.2$ and $£ 7.5$, respectively. The WTP to go from small to medium and small to high quantity of fish was $£ 6.7$ and $£ 7.7$ respectively. Anglers also preferred to fish in stillwaters rather than in rivers and canals and in sites where they can use multimethod fishing methods, rather than fly-fishing only. Most of the standard deviations of the coefficients were significant, confirming the existence of unobserved variation in the preferences of different anglers.

The WTP values associated with most fish species were negative, which means that, on average, anglers preferred sites without that species. This may be explained by the fact that anglers target a given species and attach positive value to that species and negative value to other species. The average WTP across all anglers can therefore be negative. This hypothesis was confirmed in further analysis (not shown in the paper) segmenting results according to 
the species the participants' fished in their base trip. We found that in general, participants attached positive WTP to that fish species and negative WTP to many of the other fish species.

Table 2: SP1 site choice: mixed logit model

\begin{tabular}{|c|c|c|c|c|c|c|c|}
\hline & \multicolumn{2}{|c|}{ Mean } & \multicolumn{2}{|c|}{ Std.Dev. } & \multicolumn{2}{|c|}{ WTP $(\mathfrak{f} /$ trip $)$} \\
\hline & & Coeff. & SE & Coeff & SE & Central & (95\% C.I.) \\
\hline \multicolumn{2}{|c|}{ Distance ('00 miles) } & -0.847 & $0.017^{* * *}$ & & & & \\
\hline \multicolumn{2}{|l|}{ Cost $(\mathfrak{£})$} & -0.024 & $0.001^{* * *}$ & & & & \\
\hline \multirow[t]{2}{*}{ Water body type } & River & -0.158 & $0.027^{* * *}$ & 0.974 & $0.028^{* * *}$ & -8.7 & $(-9.8,-7.7)$ \\
\hline & Canal & -0.430 & $0.062^{* * *}$ & 1.401 & $0.082^{* * *}$ & -19.0 & $(-20.2,-17.9)$ \\
\hline Method & Fly-fishing & -0.260 & $0.032^{* * *}$ & 1.359 & $0.038^{* * * *}$ & -12.7 & $(-14.2,-11.2)$ \\
\hline \multirow[t]{9}{*}{ Fish species } & Wild trout & -0.585 & $0.025^{* * *}$ & 0.388 & $0.042^{* * * *}$ & -24.9 & $(-25.2,-24.5)$ \\
\hline & Stocked trout & -0.498 & $0.027^{* * *}$ & 0.621 & $0.037^{* * *}$ & -21.4 & $(-22.0,-20.7)$ \\
\hline & Grayling & -0.286 & $0.041^{* * *}$ & 0.560 & $0.063^{* * *}$ & -12.1 & $(-12.6,-11.6)$ \\
\hline & Salmon/sea trout & -0.675 & $0.032^{* * *}$ & 0.351 & $0.097^{* * *}$ & -28.7 & $(-29.0,-28.4)$ \\
\hline & Mixed coarse & -0.090 & $0.024^{* * *}$ & 0.613 & $0.029^{* * * *}$ & -2.4 & $(-3.0,-1.8)$ \\
\hline & Predators & -0.333 & $0.025^{* * *}$ & 0.631 & $0.027^{* * *}$ & -14.1 & $(-14.7,-13.5)$ \\
\hline & Barbel & -0.101 & $0.030^{* * *}$ & 0.587 & $0.044^{* * * *}$ & -4.2 & $(-4.8,-3.7)$ \\
\hline & Carp & 0.150 & $0.035^{* * *}$ & 1.144 & $0.038^{* * * *}$ & 6.5 & $(5.2,7.7)$ \\
\hline & Catfish & -0.894 & $0.061^{* * *}$ & 0.832 & $0.097^{* * *}$ & -37.3 & $(-37.9,-36.6)$ \\
\hline \multirow[t]{2}{*}{ Size } & Medium & 0.102 & $0.016^{* * *}$ & 0.118 & $0.026^{* * *}$ & 4.2 & $(4.1,4.3)$ \\
\hline & Large & 0.177 & $0.016^{* * *}$ & 0.284 & $0.034^{* * *}$ & 7.5 & $(7.0,7.9)$ \\
\hline \multirow[t]{2}{*}{ Quantity } & Medium & 0.161 & $0.015^{* * *}$ & 0.043 & 0.026 & 6.7 & $(6.7,6.8)$ \\
\hline & High & 0.184 & $0.015^{* * *}$ & 0.035 & 0.035 & 7.7 & $(7.6,7.8)$ \\
\hline \multicolumn{2}{|c|}{ Would not choose any of the sites } & -1.461 & $0.055^{* * *}$ & 1.450 & 0.051 & & \\
\hline \multirow{3}{*}{\multicolumn{2}{|c|}{$\begin{array}{l}\text { Number of participants } \\
\text { Choice situations per participant } \\
\text { McFadden's } \mathbf{R}^{2}\end{array}$}} & & & & 3,224 & & \\
\hline & & & & & 8 & & \\
\hline & & & & & 0.22 & & \\
\hline \multicolumn{2}{|l|}{ Count $\mathbf{R}^{2}$} & & & & 0.57 & & \\
\hline
\end{tabular}

Notes: Significance levels: ${ }^{*} 10 \%,{ }^{* *} 5 \%,{ }^{* * *} 1 \%$. Omitted categories: stillwater, multimethod, small size, low quantity. Coeff: Coefficient, SE: Standard error, WTP: Willingness to pay, CI: Confidence interval. Goodness of fit statistics: McFadden $\mathrm{R}^{2}$ is the percentage reduction in the log-likelihood for the final model compared with the intercept-only model. Count $\mathrm{R}^{2}$ is the percentage of correct predictions.

\subsection{SP2: Importance of site attributes}

\section{Design}

The SP2 exercise asked participants about the most and least important attributes of fishing sites. We used the 'MaxDiff' approach (Louviere et al. 2015), which deals effectively with large numbers of attributes and it requires attributes to be described in terms of two levels only. Hence, all the attributes were described in a single phrase, with an implied counterfactual. Participants were shown eight questions (see an example in Figure 2) and asked to indicate the most important and least important attribute from a list of four attributes, in the context of a fishing occasion. The attributes were described in a single phrase, with an implied counterfactual. It was possible to choose that none of the attributes were important. 
Figure 2: SP2 question format

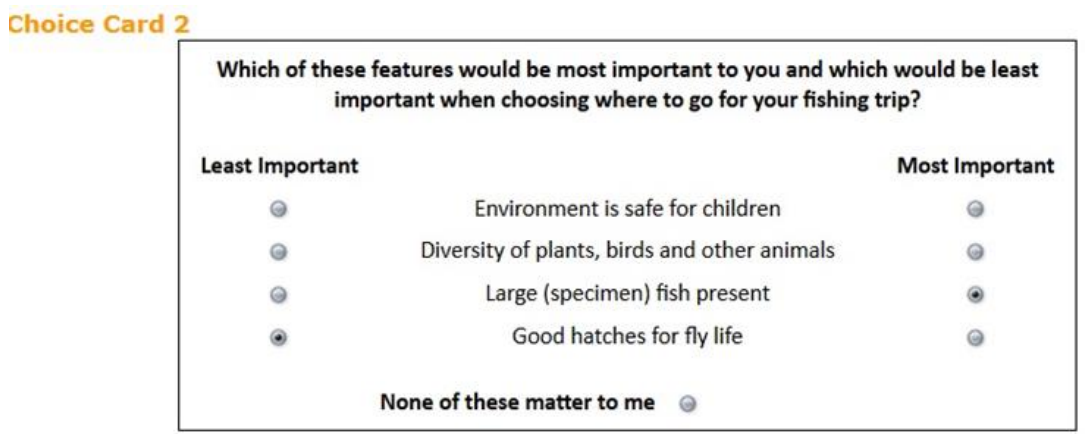

The dataset was exploded so that each choice situation was split in three separate implicit choices : one indicating that the most important attribute was chosen from a choice set formed by the four attributes and the 'none of these' option, and the other two indicating that each of the middle two attributes (not the most or least important) was individually preferred to the least important attribute in a pairwise comparison.

Table 3 shows the SP2 attributes. Two of the attributes (fish size and quantity) are common to the SP1 exercise and are used to combine the results of both exercises. The experimental design generated all possible combinations of four different attributes. These combinations were then grouped into 18 blocks so that each participant saw only a subset of the combinations. Taking all responses, this approach provides a good quality dataset for measuring the relative importance of each of the attributes.

Table 3: SP2 attributes

\begin{tabular}{ll}
\hline Attribute & Description \\
\hline Fish size & Large (specimen) fish present \\
Fish quantity & High abundance of target species - exceptional catches common \\
Litter & Site is free of litter \\
Pollution & No visible pollution \\
Pegs & Good availability of fishing spots and/or pegs at site \\
Crowding & Very few other anglers \\
Disturbance & Lack of disturbance from other site users (e.g. boating or cycling) \\
Accessibility & Good footpaths for easy access to fishing spot \\
Limited Parking & Free car park available near the water, with max stay of 3 hours \\
Unlimited Parking & Free car park available near the water, with no time limits \\
Toilets & Public toilet available at or near site \\
Plants and wildlife & Diversity of plants, birds and other animals \\
Methods & All legal fishing methods permitted, i.e. no restrictions. \\
Flies & Good hatches for fly life \\
Take & (Limited) catch can be taken away, rather than catch and release \\
Safety & Environment is safe for children \\
Crime & A very low crime rate \\
Environment & A beautiful or attractive environment \\
\hline
\end{tabular}




\section{Model}

The answers were modelled using a mixed logit specification. The utility $U_{i y}$ of attribute $y$ for angler $i$ is a function of a parameter $\phi_{i y}$, assumed to have a random and normal distribution over the population of anglers, and a random error term $\varepsilon_{i y}$.

$$
U_{i y}=\phi_{i y}+\varepsilon_{i y}
$$

The probability that angler $i$ chooses $y$ as the most important attribute in an implicit choice situation can be expressed as the probability that the utility associated with that attribute is greater than the utility associated with any other attribute $z$ in the choice set.

$$
P\left(U_{i y}>U_{i z}\right)=P\left(\phi_{i y}+\varepsilon_{i y}>\phi_{i z}+\varepsilon_{i z}\right)
$$

If the error terms are independently and identically distributed with a Type I Extreme Value distribution, then the probability $P_{i y}$ that angler $i$ chooses attribute $y$ can be expressed as below. The parameters of the model can be estimated via maximum simulated likelihood.

$$
P_{i y}=\exp \left(\phi_{i y}\right) /\left(\exp \left(\phi_{i y}\right)+\Sigma_{z} \exp \left(\phi_{i z}\right)\right)
$$

The dependent variable in the model was a dummy variable equal to 1 if the attribute was chosen by the participant as their choice and 0 otherwise. The explanatory variables were dummy variables identifying the attributes and the "none of these matters" option. The omitted attribute was "beautiful or attractive environment". All the explanatory variables were specified as having random normally distributed coefficients.

An odds ratio can be calculated for each attribute, given by the exponential of the respective model coefficient, and representing the odds than an angler will choose that attribute as the most important in an implicit choice situation, comparing with the odds of choosing the "beautiful and attractive environment" attribute.

The WTP for the SP2 attributes can be estimated by linking the SP2 and SP1 models, as they have common attributes (Ben-Akiva and Morikawa 1990, Adamowicz et al. 1994). The common attributes are fish size and quantity. We defined a scaling factor (SF) in equation (7) as the average of two ratios: 1) the ratio of the difference between the high and medium quantity coefficients in the SP1 model to the odds ratio of the quantity attribute in the SP2 model $\left(O R_{\text {quant }}\right)$; and 2$)$ the ratio of the difference between the large and medium size coefficients in the SP1 model to the odds ratio of the size attribute in the SP2 model $\left(O R_{\text {size }}\right)$. The WTP for an attribute $y$ was then calculated in equation (8) by multiplying the odds ratio of that attribute by the scaling factor and dividing by the SP1 cost coefficient $\lambda$.

$$
S F=0.5\left(\beta_{\text {highquant }}-\beta_{\text {medquant }}\right) / O R_{\text {quant }+} 0.5\left(\beta_{\text {largesize }}-\beta_{\text {medsize }}\right) / O R_{\text {size }}
$$




$$
W T P_{y}=-O R_{y} * S F / \lambda
$$

We also defined a confidence interval for WTP, with the limits derived by applying equation (8) to the limits of the confidence interval of the odds ratios. This is an imperfect solution because it assumes the scaling factor is fixed. However, taking into account the randomness of the coefficients that integrate the scaling factor equation in (7) would require complex procedures for the simulation of WTP (Espino et al. 2006), which is computationally demanding, given the size of our dataset.

\section{Results}

Table 4 shows the mean and standard deviations of the individual-level SP2 coefficients, the odds ratios, and the WTP central values and 95\% confidence interval for each attribute. The most important attributes were good availability of fishing spots/pegs, no visible pollution, and a beautiful and attractive environment and the least important were the possibility of taking catch away and the presence of good hatches for fly life. These last results may be explained by the predominance in the sample of participants who fished for coarse fish in their base trips and by the low proportion using fly-fishing in that trip. There was significant variation in preferences for almost all the attributes, as shown in the significance of the coefficients' standard deviations. The WTP estimates varied from $£ 0.3 /$ trip (for taking catch away) to $£ 6.6 /$ trip (for the availability of fishing spots/pegs). 
Table 4: SP2 MaxDiff mixed logit model

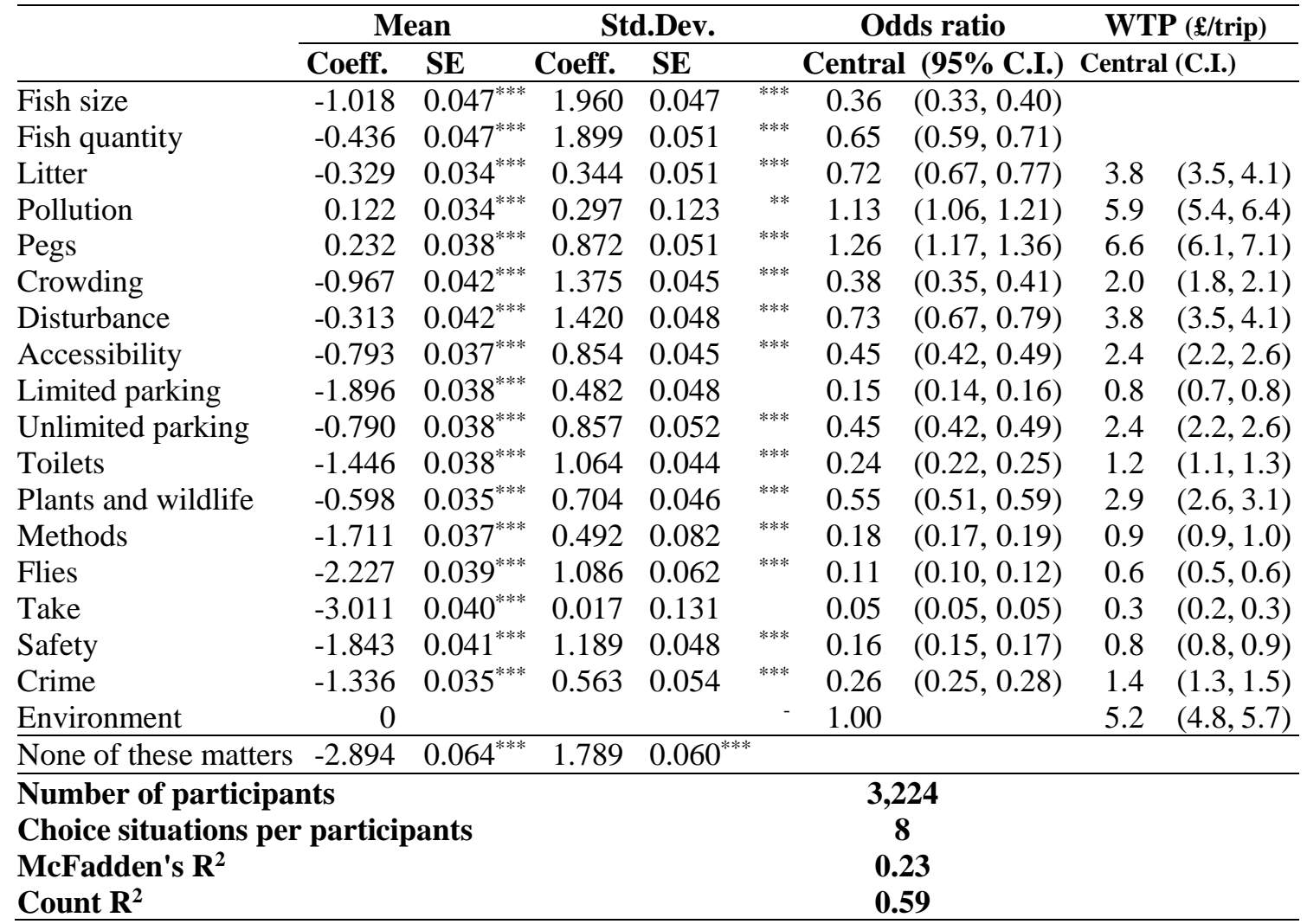

Notes: Significance levels: ${ }^{*} 10 \%,{ }^{* *} 5 \%,{ }^{* * *} 1 \%$. Omitted attribute: Environment. Coeff: Coefficient, SE: Standard error, WTP: Willingness to pay, CI: Confidence interval. For interpretation of goodness of fit statistics see Table 2.

\section{Revealed preferences}

\subsection{Data}

The second stage of the research was to estimate an RP model to examine the determinants of anglers' choice of sites and trip frequency based on real-world angler behaviour. The approach used was to collect information about sites visited by anglers (including location), build a choice set of alternative sites containing similar information, and then model the choices of sites and the number of annual trips of each angler. The distance coefficient of the site choice model was used to link the RP and SP analyses as described later in Section 6.

The data on the sites visited was obtained from an online survey of 10,468 anglers, sampled from the Environment Agency's database of rod licence holders mentioned in Section 4. The data collected in the survey includes, for each participant, the total number of days fished in 2015 for each of three types of fish (coarse fish/eels, trout/grayling, and salmon/sea trout), the locations of up to 20 visits for each of those types of fish (identified using an interactive mapping interface), and the number of days anglers fished in each 
location. A total of 10,293 participants and 21,845 visits were retained, after excluding visits to sites outside England or with unknown water body type. Anglers made an average of 27 trips over the year. The survey questionnaire also asked information about the home location of the angler (also identified in the mapping interface), as well as age, gender, and licence type and scope.

As in the case of the SP sample, the RP sample had a predominance of men (97\%), individuals aged over 45 (78\%), holding a full licence (67\%) and an annual trout and coarse licence $(89 \%)$. The sample was not representative of the population of anglers, which has smaller proportions of individuals aged over $45(53 \%)$, holding a full licence (47\%) and a trout and coarse annual licence $(77 \%)$. A raking procedure was therefore used to match the sample-weighted totals of age, gender, and licence type and scope with the target population. The weights were then applied in the estimation of the RP model.

The data source for the available fishing sites and their characteristics was the Fishing Info dataset (http://fishinginfo.co.uk), which includes information on the water body type (river, stillwater, or canal), fishery type (coarse, game, or mixed), and whether the fishery is stocked and the site includes disabled facilities or boat hire facilities. This dataset includes 4,634 sites and is the best available data source for fishing sites in England. However, it is not exhaustive (it excludes private fisheries), has missing values (3\% to $11 \%$, depending on the variable), and does not include information on permit costs.

The Fishing Info dataset was complemented with the Water Framework Directive Cycle 2 Overall Classification dataset of surface water bodies (obtained from https://data.gov.uk), which includes information on water body type (river, stillwater, canal, or transitional) and fish class status (high, good, moderate, poor, or bad) in 2015. The data includes 4,594 sites (excluding private fisheries), 2,724 of which had missing data on fish class (including all canals and stillwaters). A Geographical Information System (GIS) was used to match Fishing Info sites to the nearest feature in the water body data, if it was of the same water body type and the distance was below $1 \mathrm{~km}$. 1,564 of the 4,634 Fishing Info sites could be matched using this method.

The locations of the sites that anglers visited were then matched to the nearest Fishing Info site of the same water body type where this was within $1 \mathrm{~km} .42 \%$ of the visits were matched using this method. Unmatched sites located very close to each other were identified as 'clusters'. These were defined as groups of sites of the same water body type where each site was less than $1 \mathrm{~km}$ from at least one of the other sites and from the cluster centroid. The 
cluster centroid was taken as a 'virtual' site, representing all the sites in the cluster. $18 \%$ of the visits were matched using this method. The remaining $40 \%$ of the visits were assumed to be made to isolated sites.

The choice set of each angler then consisted of 14,148 sites, i.e. the 4,634 Fishing Info sites, 880 cluster centres, and 8,634 isolated sites, identified using the methods above. Although cluster centres and isolated sites have missing data on site characteristics, it is still useful to include them in the analysis so that the estimation of the impact of distance on site choice (which is the main purpose of the RP analysis) used the locations of all the visited sites.

Random sampling was then used to reduce the size of the choice set and the computational power required to estimate the site choice model (Parsons and Kealy 1992, Parsons and Needelman 1992, Feather 1994). This approach does not consider individual-specific preferences about their familiar and favourite sites (Parsons and Hauber 1998, Hicks and Strand 2000, Parsons et al. 2000). However, it is a reasonable approach if we treat it as a form of "long-run analysis under the assumption that eventually anglers will be aware of all sites available to them" (Peters et al. 1995, p.1786). The alternative would be to use constrained choice sets specific to each participant (Thiene et al. 2017), which is more computationally demanding and may lead to estimate bias when used in large datasets (Li et al. 2015).

For each angler, the reduced choice set was then defined as the visited sites and a number of sites randomly sampled from the full choice set, so that the choice set had exactly 500 alternatives. An extra alternative was then included to capture the option of not visiting any site.

Travel distances were calculated in GIS, using a road network model built from publiclyavailable data (Ordnance Survey Open Roads dataset). A shortest route algorithm was implemented to calculate the shortest network distances from the home locations of all the survey participants to all Fishing Info sites, cluster centres, and isolated sites. The average and standard deviation of the one-way travel distances was 115 and 65 miles respectively.

\subsection{Methods}

The data was modelled using a nested structure that follows an approach developed by Bockstael et al. (1987). A random utility site choice model explained an angler's choice of which site to visit on a given choice occasion as a function of the site characteristics, 
including its distance from the angler's home. A participation model then explained the total number of trips taken over a year, given the sites available, as a function of angler characteristics and a term known as the 'inclusive value' (or 'log sum value'), which varies over anglers. This term was derived from the site choice model and represents the maximum expected utility gained from the set of available sites. The expectation was that, on average, the higher the inclusive value, the more often anglers would go fishing.

The site choice model used a conditional logit specification (McFadden 1974), which, unlike the mixed logit specification, assumes that coefficients are fixed across individuals. It also assumes that the relative probabilities of any two alternatives being chosen are not affected by the introduction or removal of other alternatives (a property known as the independence of irrelevant alternatives). While this is a limitation, it also ensures that the random sampling approach we used to define the choice set does not prevent the estimation of consistent model coefficients (McFadden 1978).

The utility $U_{i j}$ for an angler $i$ visiting a site $j$ on a given choice situation was defined as a function of the distance from an angler's residence to the site $\left(d_{i j}\right)$, other observed site characteristics $\left(x_{j}\right)$, and a random error term $\varepsilon_{i j}$ which accounts for all the unobserved characteristics that influence the anglers' utility. $\delta$ and the vector $\tau$ are parameters to be estimated.

$$
U_{i j}=\delta d_{i j}+\tau x_{j}+\varepsilon_{i j}
$$

The probability that angler $i$ chooses to visit site $j$, given all sites $l$ in their choice set can be expressed in terms of a logistic distribution, as below. The model parameters can be estimated via maximum likelihood.

$$
P_{i j}=\exp \left(\delta d_{i j}+\tau x_{j}\right) / \sum_{l} \exp \left(\delta d_{i l}+\tau x_{l}\right)
$$

The inclusive value $V_{i}$ of angler $i$ can be calculated as the natural logarithm of the denominator in equation (10) and represents the maximum expected utility that can be gained from the choice set, as shown by Williams (1977) and Small and Rosen (1981).

$$
V_{i}=\ln \left(\sum_{l} \exp \left(\delta d_{i l}+\tau x_{l}\right)\right)
$$

The dependent variable in the site choice model was a dummy variable equal to 1 if a site alternative was chosen and 0 otherwise. The explanatory variables were the distance from home to the site, dummy variables for the different water body types, fishery types, fish class, and whether the fishery is stocked, has boat hire facilities, or disabled facilities, and a dummy variables representing the "no site is visited" alternative. 
The participation component of the model explained the total number of trips taken over a year by an angler as a function of angler-specific variables and the inclusive value from the site choice model. As the number of trips is a non-negative integer, this variable has usually been modelled using Poisson count data models (Creel and Loomis 1992, Hausman et al. 1995) or hurdle count models (Haab and McConnell 1996, Shonkwiler and Shaw 1996). However, these models do not account for unobserved heterogeneity i.e. differences across individuals that are not captured by the independent variables. Following recent literature (Hynes et al. 2015, Breen et al. 2018), we used a negative binomial model, which accounts for heterogeneity by modelling overdispersion in the dependent variable.

The distribution of the number of trips $T$ can be expressed as in equations (12) and (13), where $\mu_{i} \eta_{i}$ is the conditional mean, which depends on individual characteristics $r_{i}$, the inclusive value $V_{i}$ and a random error term $\varepsilon_{i}$ capturing unobserved factors uncorrelated with the individual characteristics. $\xi$ and the vector $\theta$ are parameters to be estimated.

$$
\begin{gathered}
f\left(T_{i} \mid r_{i}, \eta_{i}\right)=\left(\exp \left(-\mu_{i} \eta_{i}\right)^{*}\left(\mu_{i} \eta_{i}\right)^{T i}\right) / T_{i} ! \\
E\left(T_{i} \mid r_{i}, \eta_{i}\right)=\mu_{i} \eta_{i}=\exp \left(\theta r_{i}+\xi V_{i+} \varepsilon_{i}\right), \text { where } \eta_{i=} \exp \left(\varepsilon_{i}\right)
\end{gathered}
$$

If $\eta_{i}$ follows a gamma distribution such that $E\left(\eta_{i}\right)=1$ and $\operatorname{Var}\left(\eta_{i}\right)=1 / v$, then the conditional variance can be written as:

$$
\operatorname{Var}\left(T_{i} \mid r_{i}\right)=\mu_{i}\left(1+\mu_{i} / v_{i}\right)
$$

If $v_{i}=v=1 / \sigma$ for all individuals and for $\sigma>0$, this becomes:

$$
\operatorname{Var}\left(T_{i} \mid r_{i}\right)=\mu_{i}\left(1+\mu_{i} / v\right)=\mu_{i}\left(1+\sigma \mu_{i}\right)
$$

Since $\mu_{i}$ and $v$ are positive, the conditional variance in equation (15) is greater than the conditional mean. $\sigma$ is known as the dispersion parameter, since an increase in $\sigma$ leads to an increase in the conditional variance.

The site choice model can also be used to estimate willingness to pay per trip (for existing trips) for changes in site attributes. We first calculated the willingness to travel longer distances (WTT) as the ratio between the attribute coefficients and the travel distance coefficient. The $95 \%$ confidence intervals for this ratio were estimated using the Krinsky Robb method. The central estimate and the limits of the confidence interval were then doubled to account for the fact that anglers make return trips to visit the sites, and then converted to monetary values using an estimated travel cost per mile.

The travel cost per mile was estimated as $£ 0.368$ and is the sum of two components, as shown in equation (16). The first component is the out-of-pocket travel cost) (OOP), estimated as $£ 0.134 /$ mile, i.e. the average of the values for petrol and diesel cars suggested by 
the Automobile Association in 2014 (https://www.theaa.com). The second component is the value of travel time (VTT), which represents the opportunity cost of the time spent travelling and was estimated as $£ 0.234 /$ mile, i.e. the ratio between $£ 11.21$ per hour (the value of nonwork and non-commuting travel time given by DFT (2015a)) and 48mph (the average speed on single carriageway roads outside urban areas, given by DFT (2015b)).

Cost $/$ mile $=O O P+V T T=£ 0.134+£ 11.21 \mathrm{ph} / 48 \mathrm{mph}=£ 0.134+£ 0.234=£ 0.368$

\subsection{Results}

Table 5 presents the RP model results. The site choice model shows that anglers prefer sites closer to their homes, as expected. They also prefer to fish in stillwaters, mixed (coarse and game) fisheries, sites with higher fish class status, and fisheries that were stocked and had boat hire or disabled facilities. In the participation model, the inclusive value has a positive and significant coefficient, showing that anglers who can gain higher utility from the choice set of available sites tend to make more fishing trips, as expected. This suggests that improvements to a site have a positive impact on participation in addition to their impact on the share of visits going to that site. Anglers in the 25-34 and 65-74 age groups and those holding a trout/coarse licence tend to make more trips per person than other anglers. The dispersion parameter is significant, which shows that the dependent variable is overdispersed and is better modelled using a negative binomial model than a Poisson model. 
Table 5: RP model

\begin{tabular}{|c|c|c|c|c|c|c|}
\hline & \multirow{2}{*}{ Coeff. } & \multirow{2}{*}{ SE } & \multirow{2}{*}{$\underset{\text { (miles/trip) }}{\text { WTT }}$} & \multicolumn{2}{|c|}{ WTP (£/trip) } \\
\hline & & & & & Central & 95\% C.I. \\
\hline \multicolumn{7}{|l|}{ Site choice model } \\
\hline \multicolumn{2}{|c|}{ One-way travel distance from home to site (miles) } & -0.066 & $0.001^{* * *}$ & & & \\
\hline \multirow[t]{4}{*}{$\overline{\text { Water body type }}$} & River & -0.104 & $0.047^{* *}$ & -3.2 & -1.17 & $(-2.20-0.16)$ \\
\hline & Canal & -1.286 & $0.083^{* * *}$ & -39.3 & -14.5 & $(-16.2-12.5)$ \\
\hline & Transitional & -0.891 & $0.262^{* * *}$ & -27.2 & -10.0 & $(-15.7-4.06)$ \\
\hline & Unknown & -1.645 & $0.064^{* * *}$ & & & \\
\hline \multirow[t]{3}{*}{ Fishery type } & Mixed (coarse and game) & 0.712 & $0.149^{* * *}$ & 21.7 & 8.00 & $(4,79,11.2)$ \\
\hline & Game & 0.373 & $0.040^{* * *}$ & * 11.4 & 4.19 & $(3.32,5.09)$ \\
\hline & Unknown & 0.679 & $0.075^{* * *}$ & & & \\
\hline \multirow[t]{3}{*}{ Fish class } & High & 0.387 & $0.134^{* * *}$ & 11.8 & 4.35 & $(1.40,7.26)$ \\
\hline & Good and moderate & 0.209 & $0.119^{*}$ & 6.4 & 2.35 & $(-0.21,4.91)$ \\
\hline & Missing & 0.659 & $0.116^{* * *}$ & & & \\
\hline \multirow{2}{*}{$\begin{array}{l}\text { Fishery is } \\
\text { stocked }\end{array}$} & Yes & 0.212 & $0.034^{* * * *}$ & 6.5 & 2.38 & $(1.67,3.15)$ \\
\hline & Unknown & 0.210 & $0.055^{* * * *}$ & & & \\
\hline \multicolumn{2}{|c|}{ Boat hire facility Yes } & 0.681 & $0.055^{* * * *}$ & 20.8 & 7.66 & $(6.45,8.86)$ \\
\hline & Unknown & 0.557 & $0.089^{* * *}$ & & & \\
\hline \multicolumn{2}{|c|}{ Disabled facility Yes } & 0.460 & $0.028^{* * *}$ & 14.0 & 5.17 & $(4.57,5.80)$ \\
\hline & Unknown & 0.130 & 0.080 & & & \\
\hline \multicolumn{2}{|c|}{ No site is visited } & -0.774 & $0.049^{* * *}$ & & & \\
\hline \multicolumn{2}{|l|}{ Number of visits } & \multicolumn{5}{|c|}{21,845} \\
\hline \multicolumn{2}{|c|}{ Number of participants } & \multicolumn{5}{|c|}{10,293} \\
\hline \multicolumn{2}{|c|}{ Alternatives per participant } & \multicolumn{5}{|c|}{501} \\
\hline \multicolumn{2}{|c|}{ Number of observations } & \multicolumn{5}{|c|}{$5,156,793$} \\
\hline \multicolumn{2}{|l|}{ Pseudo $\mathbf{R}^{2}$} & \multicolumn{5}{|c|}{0.33} \\
\hline \multicolumn{2}{|l|}{ Count $\mathbf{R}^{2}$} & \multicolumn{5}{|c|}{0.14} \\
\hline
\end{tabular}

Participation model

\begin{tabular}{llll}
\hline Inclusive value & & 0.062 & $0.025^{* *}$ \\
\hline Age & $17-24$ & 0.015 & 0.090 \\
& $25-34$ & 0.145 & $0.072^{* *}$ \\
& $35-44$ & 0.036 & 0.068 \\
& $45-54$ & 0.004 & 0.065 \\
& $55-64$ & 0.006 & 0.064 \\
& $05-74$ & 0.109 & $0.064^{*}$ \\
\hline Licence & Trout/coarse & 0.313 & $0.052^{* * *}$ \\
\hline Constant & 2.536 & $0.181^{* * *}$ \\
\hline Dispersion parameter & 1.062 & $0.014^{* * *}$ \\
\hline Number of observations & & \multicolumn{1}{c}{$\mathbf{1 0 , 2 9 3}$} \\
\hline Notes: Significance levels: ${ }^{*} 10 \%,{ }^{* *} 5 \%,{ }^{* * *} 1 \%$. Omitted categories: (site choice model) stillwater, coarse \\
fishery, fish class poor or bad; (participation model): age > 75 years, salmon and sea trout licence. Coeff: \\
Coefficient, SE: Standard error, WTT: Willingness to travel further, WTP: Willingness to pay, CI: \\
Confidence interval. For interpretation of goodness of fit statistics of the site choice model, see Table 2.
\end{tabular}

\section{Combined analysis}

\subsection{Method}

The WTP estimates presented in the previous sections were for existing trips and did not consider switching between sites and changes in the numbers of visits. However, the RP model can be used to predict changes in the choice of sites as well as in the frequency of 
visits due to changes in site characteristics. This is because an increase in the quality of one site increases the inclusive value derived from the site choice model. which in turn increases the number of predicted trips in the participation model.

In this section we estimate the impact of changes in site characteristics on the number of visits and consumer surplus. This was done by combining the SP and RP results into a site choice utility function containing all the SP1 and SP2 site attributes but with a scale adjusted to the RP model and calibrated to real-world data. The site choice utility function and the participation model were then used to predict changes in the number of visits made by the population of licence holders to all the available sites (using the Fishing Info dataset of 4,634 sites that was used in the RP analysis), and to calculate the change in consumer surplus.

The population data was extracted from the Environment Agency's database of rod licence holders mentioned in previous sections. This data includes the postcode, age, and licence type for all 931,203 adult licence holders in England. While it was theoretically possible to estimate the probabilities that each licence holder visits each of the 4,634 sites (as home locations are unique and hence their distances to all sites are also unique), this approach was computationally infeasible because it would require the calculation of a very large number of probabilities. For this reason, we grouped licence holders into the 430 catchment areas in England, using GIS data on these areas provided by the Environment Agency. For each catchment we then calculated the number of licence holders in 28 different segments, defined as combinations of 4 different licence types and 7 age groups. 


\section{Calibration}

Figure 3 shows the approach used to calibrate the RP utility function. This was a process that adjusted the utility function so that the predicted probability that a site was visited equalled the share of the site in the total number of visits made in the real world (which was approximated by the frequency of visits to each site in the RP survey described in Section $5.1)$.

Figure 3: Calibration of utility function and prediction of probabilities

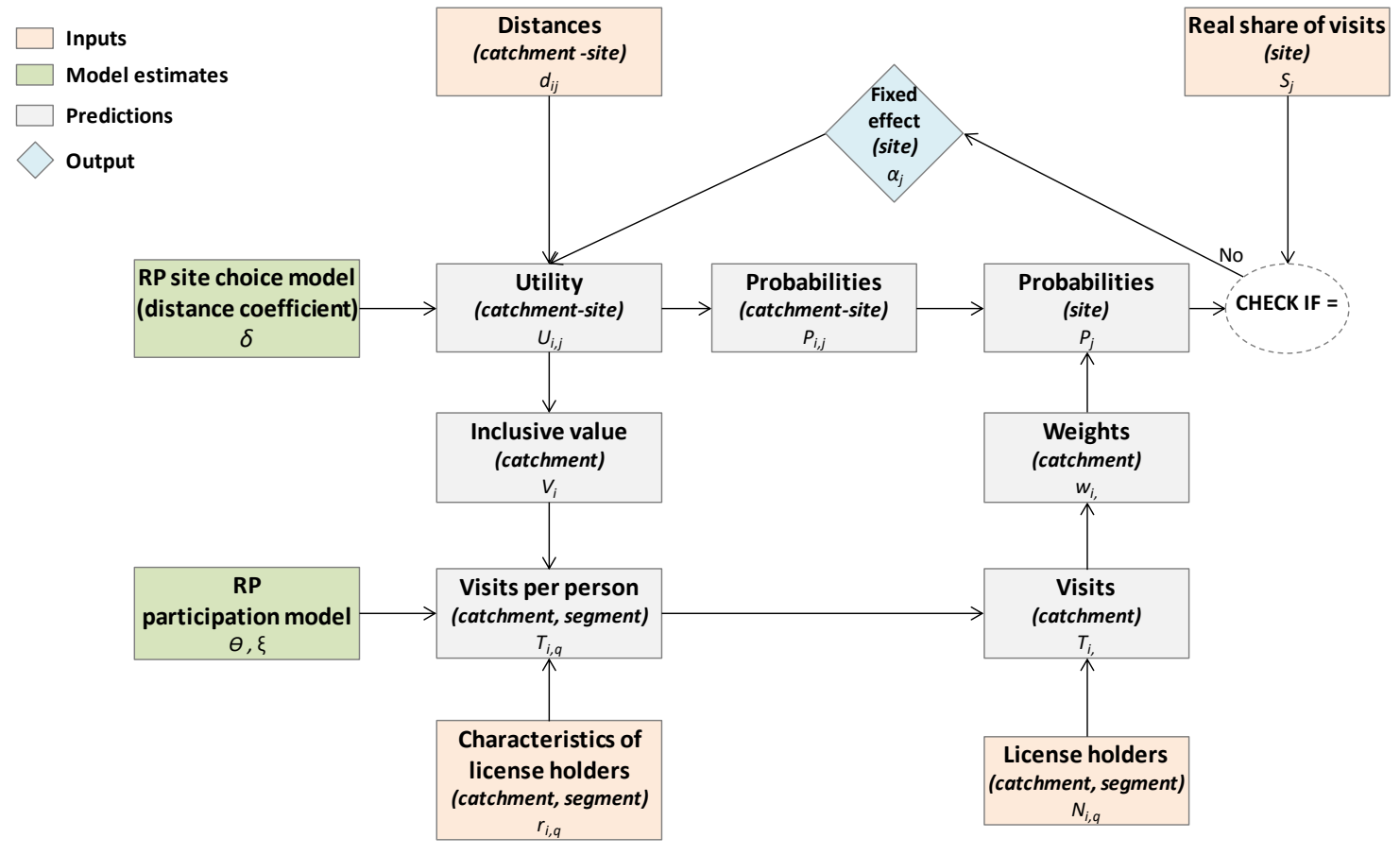

The utility $U_{i j}$ of a site $j$ for anglers living in catchment $i$ was defined as a function of the road network distance $d_{i j}$ from the population centroid of the catchment to the site (calculated using a GIS road network model) and a site fixed-effect $\alpha_{\mathrm{j}}$, initially set to 0 , accounting for the observed and unobserved characteristics of each site. The parameter $\delta$, measuring how utility varies with distance, was taken from the RP site choice model.

$$
U_{i j}=\alpha_{j}+\delta d_{i j}
$$

Assuming a logistic distribution, the predicted probability $P_{i j}$ that an angler in catchment $i$ visits site $j$ and the inclusive value $V_{i}$ of that angler can be expressed as in equations (18) and (19) respectively, where $l$ represents all the sites in the angler choice set.

$$
\begin{aligned}
P_{i j} & \left.\left.=\exp \left(U_{i j}\right)\right) /\left(\sum_{l} \exp \left(U_{i l}\right)\right)\right) \\
V_{i} & =\ln \left(\sum \exp \left(U_{i l}\right)\right)
\end{aligned}
$$


The RP participation model was then used to predict the per-angler number of annual visits to all sites $\left(T_{i, q}\right)$ made by anglers in each population segment $q$ in catchment $i$ as a function of the characteristics of that segment $r_{i, q}$, the inclusive value of the catchment $V_{i}$, and the estimated RP parameters $\theta$ and $\xi$.

$$
T_{i, q}=\exp \left(\theta r_{i, q}+\xi V_{i}\right)
$$

The total number of visits $T_{i}$ to all sites from anglers in catchment $i$ is the sum for all segments $q$, of the per-angler number of visits from anglers in that catchment and segment $\left(T_{i, q}\right)$ multiplied by the respective number of licence holders $\left(N_{i, q}\right)$.

$$
T_{i}=\sum_{q} N_{i, q} T_{i, q}
$$

The probability $P_{j}$ that an angler in any catchment visits site $j$ is then the weighted sum of the probabilities that anglers in each catchment $i$ visited that site $\left(P_{i j}\right)$, where the weight $w_{i}$ is the ratio between the visits from that catchment $T_{i}$ and the number of visits from all catchments $h$.

$$
P_{j}=\sum_{i} w_{i} P_{i j} \text { where } w_{i}=T_{i} / \sum_{h} T_{h}
$$

The model was then calibrated so that the predicted shares of each site were equal to the real shares. We applied the 'contraction algorithm' proposed by Berry et al. (1995), which is an iterative process that successively adds a quantity to the site fixed effect $\alpha_{j}$ for each site $j$ in the site choice utility function in equation (17) until the predicted shares $P_{j}$ and real shares $S_{j}$ are equalized for all sites $j$. The quantities added in each iteration $m$ were the log of the ratio between the real and the predicted shares:

$$
\alpha_{j}^{m+1}=\alpha_{j}^{m}+\log \left(S_{j} / P_{j}\right)
$$

The output of this process was a calibrated utility function, specified as in equation (17) and with a fixed effect $\alpha_{j}$ for each site.

\section{Impact of site improvements}

The calibrated site choice utility function was then used to estimate the impact of changes in the attributes of each of the 4,634 sites on the total number of visits to all sites and on the shares of each site.

For a given attribute and site, the attribute change was first converted into utility change using the SP models scaled to the RP site choice model. This was done by linking the coefficients of the attributes shared by the models. The SP2 model was first scaled to the SP1 model using the method described in Section 4.2. Both SP models were then scaled to the RP model by multiplying their coefficients by the ratio between the RP and SP1 coefficients on 
travel distance. This approach maintained all the relative values from the SP models but adjusted the scale (i.e. the extent to which site choices were driven by observed characteristics versus unobserved factors) using the RP results. The attribute change was then multiplied by the corresponding scaled coefficient to derive the corresponding utility change.

We then calculated the impact on the number of visits to all sites and on the share of each site by applying equations (18) to (22) using the initial and final values of $U_{i, j}$, i.e. the utilities of the improved site $j$ for each angler $i$ before and after the improvement. The number of visits switched to the improved site from other sites can be calculated as the difference between the change in predicted visits to the improved site and the change in the visits to all sites (since all new visits are made to the improved site).

The change in annual consumer surplus $\Delta C S_{i, j, x}$ for an angler in catchment $i$ derived from an improvement in attribute $x$ in site $j$ was then derived as the change in the utility derived by the angler from the choice set (i.e. the difference between the product of the inclusive value $V_{i}$ and the predicted number of trips $T_{i}$ after and before the improvement, noted moments 1 and 0 in the equation below) divided by the marginal utility of money $\lambda$ (i.e. the coefficient of the cost variable in the SP1 model scaled to the RP model).

$$
\Delta C S_{i, j, x}=\left(V_{i}^{l} T_{i}^{l}-V_{i}^{0} T^{0}\right) / \lambda
$$

The total change in consumer surplus for the angler population, given a change in attribute $x$ in site $j$ was then calculated the sum of the product of the per-angler change in consumer surplus in each catchment $\Delta C S_{i, j, x}$ and the number of licence holders within that catchment $N_{i}$.

$$
\triangle C S_{j, x}=\sum_{i} N_{i} \Delta C S_{i, j, x}
$$

Finally, the average change in consumer surplus across all sites of a change in attribute $x$ can be calculated as below, where $n$ is the number of sites.

$$
\Delta C S_{x}=\sum_{j} \Delta C S_{j, x} / n
$$

\subsection{Results}

Table 6 shows the predicted average impact across all sites of different types of changes in site attributes on visit numbers to the site improved and on consumer surplus. The results are expressed in relation to the number of visits before the change. It should be noted that even though consumer surplus is expressed in relation to the number of visits before the change, it incorporates benefits for three types of anglers: those who remain as visitors to the improved site, those who switch to the improved site from other sites and those who make new visits to 
the improved site. The limits of the confidence interval were approximated as the product of the central estimate by the ratio between the $95 \%$ confidence interval limits and the central estimate of the scaled SP cost coefficient.

Table 6: Predicted average impact of site improvements on number of visits and consumer surplus

\begin{tabular}{|c|c|c|c|c|c|}
\hline \multirow[t]{2}{*}{ Type of change } & \multicolumn{3}{|c|}{$\begin{array}{c}\text { Change in } \\
\text { number of visits to site } \\
\text { (as \% of existing visits) }\end{array}$} & \multicolumn{2}{|c|}{$\begin{array}{c}\text { Change in } \\
\text { consumer surplus } \\
\text { (per existing visit) }(\mathfrak{k})\end{array}$} \\
\hline & $\begin{array}{l}\text { Switched } \\
\text { visits }\end{array}$ & $\begin{array}{l}\text { New } \\
\text { visits }\end{array}$ & Total & Central & $\begin{array}{l}\text { Confidence } \\
\text { Interval }\end{array}$ \\
\hline \multicolumn{6}{|l|}{ Fish size } \\
\hline Small to Medium & 146 & 9 & 155 & 10.63 & $(10.27,10.63)$ \\
\hline Medium to Large & 104 & 6 & 110 & 7.76 & $(7.50, \quad 7.76)$ \\
\hline \multicolumn{6}{|l|}{ Fish quantity } \\
\hline Low to Medium & 331 & 18 & 349 & 22.27 & $(21.52, \quad 22.27)$ \\
\hline Medium to High & 31 & 2 & 33 & 2.43 & $(2.35,2.43)$ \\
\hline \multicolumn{6}{|l|}{ Locational characteristics } \\
\hline Litter & 123 & 8 & 131 & 9.08 & $(8.77,9.39)$ \\
\hline No visible pollution & 222 & 13 & 234 & 15.58 & $(15.05,16.11)$ \\
\hline Availability of fishing spots \&/or pegs at site & 294 & 17 & 310 & 20.04 & $(19.36,20.72)$ \\
\hline Number of other anglers & 63 & 4 & 67 & 4.80 & $(4.63,4.96)$ \\
\hline Disturbance from other site users & 125 & 8 & 133 & 9.22 & $(8.91,9.54)$ \\
\hline Public toilet & 38 & 2 & 41 & 2.97 & $(2.87,3.07)$ \\
\hline Footpaths for easy access to fishing spot & 75 & 5 & 80 & 5.71 & $(5.51,5.90)$ \\
\hline Free car park, with no time limits & 76 & 5 & 80 & 5.73 & $(5.53,5.92)$ \\
\hline Free car park, with max stay of 3 hours & 24 & 2 & 26 & 1.90 & $(1.83,1.96)$ \\
\hline All legal fishing methods permitted & 29 & 2 & 31 & 2.28 & $(2.20,2.36)$ \\
\hline Good hatches of fly life & 17 & 1 & 18 & 1.36 & $(1.32,1.41)$ \\
\hline (Limited) catch taken away & 8 & 1 & 8 & 0.62 & $(0.60,0.64)$ \\
\hline Environment is safe for children & 26 & 2 & 27 & 2.00 & $(1.93,2.07)$ \\
\hline Crime rate & 43 & 3 & 46 & 3.32 & $(3.20,3.43)$ \\
\hline Diversity of plants, birds and other animals & 93 & 6 & 98 & 6.94 & $(6.70,7.17)$ \\
\hline A beautiful or attractive environment & 176 & 10 & 186 & 12.62 & $(12.19,13.04)$ \\
\hline
\end{tabular}

The table shows that increases in fish size and quantity are predicted to cause substantial changes in the number of visits and consumer surplus, especially when moving from small/low to medium levels. For example, on average, an increase of fish quantity from low to medium would lead to a $349 \%$ increase in the number of visits to the improved site and an increase in consumer surplus of $£ 22.27$ per existing trip. This value is consistent with those from the literature. For example, Lawrence and Spurgeon (2007) found values to prevent severe declines in fish stocks of between $£ 15.80$ and $£ 23.90$ per household, depending on the method, and TDI (2013) found a value of $£ 15.97$ per person. 


\section{Discussion and conclusions}

This paper estimated the value of changes in the quality of freshwater angling sites based on the results of a large national-scale study in England. The study provides comprehensive information into what anglers value and how much, contributing to the literature by combining SP and RP data, considering a large number of attributes related to angling, and including all fishery and water body types as well as a wide range of fish species. The main output was the predicted average impact of changes in site attributes on the number of visits to the improved sites (identifying new visits and visits switched from other sites) and on consumer surplus.

The combination of SP and RP data allowed us to capture anglers' preferences over a variety of attributes, while grounding the estimates in real-world behaviour. The magnitude of the values found for improvements in the attributes that are usually included in valuation studies, such as fish size and quantity, was consistent with those found in previous studies. However, the study went beyond the available evidence, as it estimated the value of a set of locational attributes of sites, seldom included in previous studies. Some of these attributes, such as pollution, availability of pegs, and environment, were found to have a substantial value for anglers. By including all fishery and water body types, and a wide range of species, the study also captured information about preferences of anglers over these attributes, while ensuring that the preferences over the other attributes took into account the heterogeneity among anglers in terms of the fishery and water body types they visited and the species they targeted.

The predictions were calculated for a non-exhaustive group of angling sites, for which data was available. However, they can be useful as a baseline for assessments in other locations, and deployed at local, catchment and project-specific scales, and integrated into the strategic management plans of fishing resources. The results also have implications for public policies regarding the strategic management and protection of fishing resources. In particular, the high consumer surplus values found for the removal of visible pollution and the aesthetics and attractiveness of the local environment emphasize the importance of improving aspects related to the quality of the environment experienced by anglers. As these aspects also benefit the wider population, the results suggest the existence of synergies between the management of fishing resources and broader environmental policies.

The application of the results should consider a few caveats in the methods used to derive them. For example, the SP analysis did not consider the supply side, i.e. the location of 
angling opportunities relative to the population and how anglers react to these opportunities. In a region where a certain site attribute is scarce there might be a premium for this attribute. For example, in a region where most sites do not have large-sized fish, anglers may be willing to pay an extra amount for visiting sites with this attribute. In addition, increases in the number of visits to a site might have impacts on some attributes, such as crowding or the availability of pegs, which would reduce demand for that site. These aspects were only partly addressed by linking the SP and RP estimates. By combining the SP1 and SP2 results, we also assumed that participants' responses to those exercises arise from the same underlying preferences.

The RP analysis was also limited by the fact that the set of sites was not exhaustive and had missing data. The RP site choice model also did not consider some factors that influence site choice (such as cost, aesthetic appeal, and remoteness) and the participation model did not consider socio-economic variables. This may lead to bias in the model estimates if those factors are correlated with any of the variable in the model. Anglers may also have different perceptions about the same objectively measured attributes (Deely et al. 2019). As previously mentioned, the RP site choice model, which has a conditional logit specification, did not account for preference heterogeneity across participants and assumed the independence of irrelevant alternatives, which may lead to unreliable estimates if this condition does not hold. The model was also not dynamic in the sense that it did not allow for relationships between the site choices of the same anglers on different choice occasions (i.e. the choice of site on each occasion is not influenced by previous experiences).

Predicted numbers of new and switched visits may also be overestimated due to the large number of possible sites in the choice set for each angler coupled with the fact that there was no 'habit' element in the model. As such, the SP models did not capture the inertia that is often found in real-world angler behaviour, even when those models were calibrated using RP data. Our approach also assumed that all current or potential users were fully aware of the changes in a site. This issue could be addressed in future research by scaling the changes in utility in order to account for the levels of awareness about changes in each attribute amongst users. Finally, the estimated changes in number of visits referred only to the current licence holders, but it is possible that improvements in site quality may induce some people to acquire a licence and start making visits to fishing sites. Our estimates did not account for this possibility, which is a limitation, although it may also contribute to balancing the overall tendency of our approach for the overestimation of the changes in the number of visits. 


\section{Acknowledgements}

This work was supported by the UK Environment Agency.

\section{References}

Adamowicz, W., Louviere, J., Williams, M. (1994) Combining stated and revealed preference methods for valuing environmental amenities. Journal of Environmental Economics and Management 26(3), 271292.

Arlinghaus, R. (2004) Angelfischerei in Deutschland - Eine Soziale und Ökonomische Analyse [Recreational Fisheries in Germany - A Social and Economic Analysis]. Leibniz-Institute of Freshwater Ecology and Inland Fisheries, Berlin [in German].

Arismendi, I., Nahuelhual, L. (2007) Non-native salmon and trout recreational fishing in Lake Llanquihue, Southern Chile: economic benefits and management implications. Reviews in Fisheries Science 15(4), 311-325.

Berrens, R., Bergland, O., Adams, R M. (1993) Valuation issues in an urban recreational fishery - spring chinook salmon in Portland, Oregon. Journal of Leisure Research 25, 70-83.

Ben-Akiva, M E., Morikawa, T. (1990) Estimation of travel demand models from multiple data sources. Proceedings of the 11th International Symposium on Transportation and Traffic Theory, Yokohama.

Ben-Akiva, M E., Morikawa, T. (1990) Estimation of travel demand models from multiple data sources. Proceedings of the 11th International Symposium on Transportation and Traffic Theory, Yokohama.

Berry, S., Levinsohn, J., Pakes, A. (1995) Automobile prices in market equilibrium. Econometrica 63(4), 841-890.

Bockstael, N E., Hanemann, W M., Kling, C L. (1987) Estimating the value of water quality improvements in a recreational demand framework. Water Resources Research 23(5), 951-960.

Breen, B., Curtis, J., Hynes, S. (2018) Water quality and recreational use of public waterways. Journal of Environmental Economics and Policy 7(1), 1-15.

Brown, A. (2014) Realising the Eden's Angling Potential. Stage Two: Angler Survey. Report to Eden Rivers Trust, River Eden and District Fisheries Association, and Environment Agency., http://www.edenfishing.co.uk/docs/survey2014final.pdf

Butler, J R A., Radford, A., Riddington, G., Laughton, R. (2009) Evaluating an ecosystem service provided by Atlantic salmon, sea trout and other fish species in the River Spey, Scotland: the economic impact of recreational rod fisheries. Fisheries Research 96(2-3), 259-266.

Creel, M., Loomis, J. (1992) Recreation value of water to wetlands in the San Joaquin Valley: linked multinomial logit and count data trip frequency. Water Resources Research 28(10), 2597-2606.

Curtis, J A. (2002) Estimating the demand for salmon angling in Ireland. Economic and Social Review 33(3), 319-322.

Curtis, J., Breen, B. (2017) Irish coarse and game anglers' preferences for fishing site attributes. Fisheries Research 190, 103-112. 
Curtis, J., Stanley, B. (2016) Water quality and recreational angling demand in Ireland. Journal of Outdoor Recreation and Tourism 14, 27-34.

Deely, J., Hynes, S., Curtis, J. (2019) Are objective data an appropriate replacement for subjective data in site choice analysis? Journal of Environmental Economics and Policy 8(2), 159-178.

DFT (Department for Transport) (2015a) Provision of Market Research for Value of Travel Time Savings and Reliability. Non-technical Summary Report., https://www.gov.uk/government/publications/valuesof-travel-time-savings-and-reliability-final-reports

DFT (Department for Transport) (2015b) Free Flow Vehicle Speed Statistics in Great Britain: 2015 report., https://www.gov.uk/government/statistics/free-flow-vehicle-speeds-in-great-britain-2015

Eom, Y-S., Larson, D M. (2006) Improving environmental valuation estimates through consistent use of revealed and stated preference information. Journal of Environmental Economics and Management 52(1), 501-516.

Espino, R., Ortúzar, J D., Román, C. (2006) Confidence interval for willingness to pay measures in mode choice models. Networks and Spatial Economics 6(2), 81-96.

Feather, P. (1994) Sampling and aggregation issues in random utility model estimation. American Journal of Agricultural Economics 76(4), 772-780.

Haab, T., McConnell, K E (1996) Count data models and the problem of zeros in recreation demand analysis. American Journal of Agricultural Economics 78(1), 89-102.

Hanemann, M. (1984) Discrete/continuous models of consumer demand. Econometrica 52(3), 541-561.

Hausman, J A., Leonard, G K., McFadden, D. (1995) A utility-consistent, combined discrete choice and count data model - assessing recreational use losses due to natural resource damage. Journal of Public Economics 56(1), 1-30.

Hicks, R L., Strand, I E. (2000) The extent of information: its relevance for random utility models. Land Economics 76(3), 374-385.

Hughes RM (2015) Recreational fisheries in the USA: economics, management strategies, and ecological threats. Fisheries Science 81, 1-9.

Hynes, S., O'Reilly, P., Corless, R. (2015) An on-site versus a household survey approach to modelling the demand for recreational angling: do welfare estimates differ? Ecosystem Services 16, 136-145.

Johnstone, C., Markandya, A. (2006) Valuing river characteristics using combined site choice and participation travel cost models. Journal of Environmental Management 80(3), 237-247.

Kott, P S. (2009) Calibration weighting: combining probability samples and linear prediction models. Handbook of Statistics 29(B), 55-82.

Krinsky I., Robb A L. (1986) On approximating the statistical properties of elasticities. Review of Economics and Statistics 68(4), 715-719.

Laitila, T., Paulrud, A. (2006) A multi-attribute extension of discrete-choice contingent valuation for valuation of angling site characteristics. Journal of Leisure Research 38(2), 133-142. 
Lawrence, K S., Spurgeon, J. (2007) Economic Evaluation of Inland Fisheries: Welfare Benefits of Inland Fisheries in England \& Wales. Environment Agency, Bristol., https://www.gov.uk/government/uploads/system/uploads/attachment data/file/291114/sw2-039-pr-1-ee.pdf

Lee, D E., Hosking, S G., Preez M. (2013) A choice experiment application to estimate willingness to pay for controlling excessive recreational fishing demand at the Sundays River Estuary, South Africa. Water SA 44(1)., https://www.ajol.info/index.php/wsa/article/view/99662

Li, L., Adamowicz, W., Swait, J. (2015) The effect of choice set misspecification on welfare measures in random utility models. Resource and Energy Economics 42, 71-92.

Louviere, J., Flynn, T., Marley, A. (2015) Best-worst Scaling: Theory, Methods and Applications. Cambridge University Press, Cambridge.

McFadden, D. (1974) Conditional logit analysis of qualitative choice behavior., in P Zarembka (Ed.) Frontiers in Econometrics. Academic Press, New York., pp.105-42.

McFadden, D. (1978) Modelling the choice of residential location., in A Karlqvist, L Lundqvist, F Snickars and J Weibull (Eds.) Spatial Interaction Theory and Planning Models. North-Holland, Amsterdam., pp. 75-96.

McFadden, D., Train, D. (2000) Mixed mnl models of discrete response. Journal of Applied Econometrics 15(5), 447-470.

Melstrom, R T., Lupi, F., Esselman, P C., Stevenson, R J. (2015) Valuing recreational fishing quality at rivers and streams. Water Resources Research 51., doi:10.1002/2014WR016152

Mkwara, L., Marsh, D., Scarpa, R. (2015) The effect of within-season variability on estimates of recreational value for trout anglers in New Zealand. Ecological Economics 119, 338-345.

Morey, E R., Breffle, W S., Rowe, R D., Waldman, D M. (2002) Estimating recreational trout fishing damages in Montana's Clark Fork River basin: summary of a natural resource damage assessment. Journal of Environmental Management 66(2), 159-170.

Murdock, J. (2006) Handling unobserved site characteristics in random utility models of recreation demand. Journal of Environmental Economics and Management 51(1), 1-25.

Parsons, G., Hauber, A. (1998) Spatial boundaries and choice set definition in a ramdom utility model of recreation demand. Land Economics 74(1), 32-48.

Parsons, G., Kealy, M J. (1992) Randomly drawn opportunity sets in a random utility model of lake recreation. Land Economics 68(1), 93-106.

Parsons, G., Massey, D., Tomasi, T. (2000) Familiar and favorite sites in a random utility model of beach recreation. Marine Resources Economics 14(4), 299-315.

Parsons, G., Needelman, M S. (1992) Site aggregation in a random utility model of recreation. Land Economics 68(4), 418-433.

Paulrud, A., Laitila, T. (2013) A cost benefit analysis of restoring the Em River in Sweden: valuation of angling site characteristics and visitation frequency. Applied Economics 45(16), 2255-2266. 
Peirson, G., Tingley, D., Spurgeon, J., Radford, A. (2001) Economic evaluation of inland fisheries in England and Wales. Fisheries Management and Ecology 8(4-5), 415-424.

Peters, T., Adamowicz, W L., Boxall, P C. (1995) Influence of choice set considerations in modeling the benefits from improved water quality. Water Resources Research 31(7), 1781-1787.

PWC (Price Waterhouse Coopers) (2007) The Social and Economic Impact to Northern Ireland, and Areas within the Loughs Agency, of Recreational Fisheries, Angling and Angling Resources. Report to the Department of Culture, Arts and Leisure, The Loughs Agency of the Foyle, Carlingford and Irish Lights Commission and the Northern Ireland Tourist Board., https://www.daera-ni.gov.uk/publications/socialand-economic-impact-angling-and-angling-resources-final-report

Radford, A., Riddington, G., Gibson, H. (2007) Economic Evaluation of Inland Fisheries: The Economic Impact of Freshwater Angling in England \& Wales. Environment Agency, Bristol., https://www.gov.uk/government/uploads/system/uploads/attachment data/file/291117/scho1207bnnw-ee.pdf

Särndal, C-E (2007) The calibration approach in survey theory and practice. Survey Methodology 33(2), 99-119.

Shonkwiler, J S., Shaw, W D. (1996) Hurdle count-data models in recreation demand analysis. Journal of Agricultural and Resource Economics 21(2), 210-219.

Shrestha, R K., Seidl, A F., Moraes, A S. (2002) Value of recreational fishing in the Brazilian Pantanal: a travel cost analysis using count data models. Ecological Economics 42(1-2), 289-299.

Simpson, D., Mawle, G W. (2010) Public Attitudes to Angling. Environment Agency, Bristol., http://resources.anglingresearch.org.uk/sites/resources.anglingresearch.org.uk/files/EA_Public_Attitudes _to_Angling_2010.pdf

Small, K., Rosen, H. (1981) Applied welfare economics of discrete choice models. Econometrica 49(1), 105-130.

SQW (2015) Economic Impact from Angling on the Tweed River System. Report to River Tweed Commission., https://rtc.org.uk/2015_Tweed_impact_update_final_report_161115.pdf

TDI (Tourism Development International) (2013) Socio-economic Study of Recreational Angling in Ireland. Report to Inland Fisheries Ireland., http://www.fisheriesireland.ie/media/tdistudyonrecreationalangling.pdf

Thangavelu, T., Paulrud, A., Stage, J. (2017) Understanding heterogeneous preferences for angling site attributes: application of a choice experiment. Journal of Environmental Economics and Policy 6(3), 324-340.

Théberge, A. (2000) Calibration and restricted weights. Survey Methodology 26(1), 99-107.

Thiene, M., Swait, J., Scarpa, R. (2017) Choice set formation for outdoor destinations: the role of motivations and preference discrimination in site selection for the management of public expenditures on protected areas. Journal of Environmental Economics and Management 81, 152-173. 
Toivonen, A.L., Roth, E., Navrud, S., Gudbergsson, G., Appelblad, H., Bengtsson, B., Tuunainen, P. (2004) The economic value of recreational fisheries in Nordic countries. Fisheries Management and Ecology 11(1), 1-14.

Train, K E. (1998) Recreation demand models with taste differences over people. Land Economics 74(2), 230-239.

Wallentin, E. (2016) Choice of the angler: estimating single-site recreation demand using revealed preference data. Tourism Economics 22(6), 1338-1351.

Whitehead, J C. (2005) Combining willingness to pay and behavior data with limited information. Resource and Energy Economics 27(2), 143-155.

Whitehead, J C., Lee, D K., (2016) Estimating recreation benefits through joint estimation of revealed and stated preference discrete choice data. Appalachian State University Department of Economics Working Paper 16-22.

Williams, H. (1977) On the formation of travel demand models and economic evaluation measures of user benefits. Environment and Planning A 9, 285-344.

Winfield, I J. (2016) Recreational fisheries in the UK: natural capital, ecosystem services, threats, and management. Fisheries Science 82(2), 203-212. 